\title{
TCHEBYCHEV NETS ON SPHERES
}

\author{
BY \\ SANDRA LEE SAMELSON \\ State University of New York, College at New Paltz, New Paltz, New York
}

\begin{abstract}
We show that the standard sphere on $\mathbb{R}^{3}$ admits a Tchebychev net which covers an open subset of the sphere which contains a closed hemisphere.
\end{abstract}

1. Introduction. Tchebychev introduced the notion of what are now called Tchebychev nets in [Tch] in order to model the deformation of cloth. In this model a surface is considered to consist of two families of "fibers" which are inextensible, although the angle between fibers belonging to the two families can vary. Moreover this surface is assumed to be initially planar and to have obtained the current shape after going through a deformation which did not change the lengths of the fibers. The simplest way to describe such a surface is to say that it admits a coordinate chart such that the coordinate vector fields have unit magnitudes with respect to the ambient Euclidean metric.

Bieberbach [Bie] proved that every surface is locally a Tchebychev net, i.e., around every point there exists a coordinate chart such that coordinate vector fields have unit magnitudes. This was accomplished by reducing the problem to that of existence of solutions to a pair of quasilinear hyperbolic equations. However, the global issue, i.e., existence of a Tchebychev net over a prescribed open set has not adequately been investigated, except for an important equation called Hazzidaki's formula, which was first derived in [Haz] (see [St], [Pi], etc.) which shows that at least on a simply connected, open surface which is complete with respect to its Riemannian metric, the integral of the Gauss curvature must be less than $2 \pi$ in magnitude in order for a Tchebychev net to cover the whole surface. For example there does not exist a Tchebychev net covering a semi-infinite cyclinder having one end closed.

One important question is whether we can find a Tchebychev net on the standard sphere. It is easy to show that the open hemisphere and the sphere with north and south poles removed admits global Tchebychev nets. Tchebychev claimed that a hemisphere can be clothed in its entirety [Tch]. The purpose of this paper is to show that there does exist a Tchebychev net which covers a closed hemisphere. Our proof goes as follows. It is well known ([St], [Pi], etc.) that if $x_{1}, x_{2}$ denote the coordinates along the fibers of a Tchebychev net and if $\kappa$ and $\alpha$ denote the Gauss curvature and the angle between fibers, respectively, then $\partial^{2} \alpha / \partial x_{1} \partial x_{2}=-\kappa \sin \alpha$. 
In our case $\kappa \equiv 1$ and hence we have the sine-Gordon equation. We use a particular solution of the sine-Gordon equation and put a Riemannian metric of the form $d x_{1}^{2}+2 \cos \alpha d x_{1} d x_{2}+d x_{2}^{2}$ on some open subset of $\mathbb{R}^{2}$ and construct an isometry from a subset of the standard sphere onto this chart. By using careful estimates we show that the domain of this chart contains a closed hemisphere and hence we arrive at the stated conclusion.

Although it was generally believed that a Tchebychev net covering the closed hemisphere exists, as far as we know, ours is the first rigorous mathematical proof of the existence of such a net.

2. Preliminaries and the main result. Let $S^{2}$ denote the unit sphere in $\mathbb{R}^{3}$ endowed with the Riemannian metric $g$ induced from the Euclidean metric in $\mathbb{R}^{3}$.

Definition 2.1. A Tchebychev net on $S^{2}$ is a coordinate chart $(U,(x, y))$ in $\mathbb{R}^{2}$ such that the coordinate vector fields $\left(\frac{\partial}{\partial x}, \frac{\partial}{\partial y}\right)$ have unit magnitudes with respect to $g$. These vector fields will be called fiber fields and thier integral curves will be called fibers.

Theorem 2.2 (MAIN Theorem). There exists a Tchebychev net $(U,(x, y))$ on $S^{2}$ such that $U$ contains a closed hemisphere.

3. Proof of the Main Theorem. Our aim is to construct a Tchebychev net on $S^{2}$ such that orthogonal longitudes through the south pole are fibers. Let $(U,(x, y))$ be a Tchebychev net on $S^{2}$. Define $\gamma: U \rightarrow \mathbb{R}$ such that $\gamma(p)$ is the angle betwen $\left.\frac{\partial}{\partial x}\right|_{p}$ and $\left.\frac{\partial}{\partial y}\right|_{p}$. Hence $\gamma=\frac{\pi}{2}$ at the south pole. It is well known that $\gamma$ is related to the Gauss curvature $\kappa$ (in our case $\kappa \equiv 1$ ) by the relation [Pi], $\partial^{2} \gamma / \partial x \partial y=-\kappa \sin \gamma$. Also $\frac{\partial}{\partial x}$ is parallel along the integral curves of $\frac{\partial}{\partial y}$ and vice versa (in the sense of Levi-Civita) and this forces $\gamma$ to be $\frac{\pi}{2}$ on each of the two longitudes mentioned above. Therefore we are led to the characteristic initial value problem

$$
\frac{\partial^{2}}{\partial x \partial y}=-\sin \gamma, \quad \gamma(x, 0)=\frac{\pi}{2}=\gamma(0, y)
$$

for $x, y \in[0, a]$, where $a$ is positive.

To simplify the equations let

$$
\alpha:=\frac{\pi}{2}-\gamma
$$

Then

$$
\begin{aligned}
\frac{\partial^{2} \alpha}{\partial x \partial y} & =\cos \alpha(x, y), \\
\alpha(x, 0) & =0=\alpha(0, y) .
\end{aligned}
$$

Equivalently,

$$
\alpha(x, y)=\int_{0}^{x} \int_{0}^{y} \cos (\alpha(x, y)) d x d y .
$$

It is well known that Eq. (3.3) admits a unique solution on $\mathbb{R}^{2}$ and the solution depends only on the product of $x$ and $y$. One way to establish this is to assume a 
solution of the form $\alpha=\alpha(x y)$ and rewrite (3.3) in the form,

$$
t \frac{d^{2} \alpha}{d t^{2}}+\frac{d \alpha}{d t}=\cos a(t), \quad \alpha(0)=0
$$

where $t=x y$.

The existence of a solution can be shown by assuming a power series for $\alpha$ and obtaining unknown coefficients by equating coefficients in (3.5). However we need some sharp estimates on the solution and therefore we work with (3.4) and find a solution by iterating the right-hand side. Since this technique is standard, we will omit the details and list only the relevant facts. The estimates given below are obtained by using the contraction mapping property.

Let $a>0$ and let

$$
J_{a}:=[-a, a] \times[a, a] .
$$

FACT 3.1. For arbitrary $a>0,(3.4)$ admits a unique $C^{\infty}$-solution. The algorithm,

$$
\begin{gathered}
\alpha_{0}(x, y)=0, \\
\alpha_{\kappa+1}(x, y)=\int_{0}^{x} \int_{0}^{y} \cos \left(\alpha_{\kappa}(\theta, \phi)\right) d \phi d \theta, \quad j \geq 0,(x, y) \in J_{a},
\end{gathered}
$$

yields a sequence of continuous functions $\left\{\alpha_{j}\right\}_{j=0}^{\infty}$ on $J_{a}$ which converges uniformly to the solution of (3.4).

FACT 3.2. The solution $\alpha \in C^{\infty}\left(J_{a}\right)$ depends only on $(x y)$ and $x y \alpha(x y) \geq 0$ for all $(x, y) \in J_{a}$.

FACT 3.3. If $a<4$, then

$$
\left|\left(\alpha-\alpha_{2}\right)(x, y)\right| \leq \frac{|x y|^{3}}{(3 !)^{2}} \frac{1}{\left(1-|x y| / 4^{2}\right.} \quad \text { for all }(x, y) \in J_{a} .
$$

This then yields

$$
\left|\alpha(x, y)-x y-\frac{x^{3} y^{3}}{18}\right| \leq\left|\frac{2\left(x^{3} y^{3}\right)}{63}+\frac{x^{5} y^{5}}{300}\right|
$$

for all $(x, y) \in \mathbb{R}^{2}$ such that $|x y|<\frac{\pi}{2}$. In particular $|\alpha(x, y)|<\frac{\pi}{2}$ for all $(x, y) \in$ $\mathbb{R}^{2}$ such that $|x y|<\frac{\pi}{2}$. Now let $\mathscr{D}=\left\{(x, y) \in \mathbb{R}^{2}:|x|<\pi,|y|<\pi,|x y|<\frac{\pi}{2}\right\}$ and let $\gamma=\frac{\pi}{2}-\alpha$ on $\mathscr{D}$. Then $\gamma$ satisfies

$$
\frac{\partial^{2} \gamma}{\partial x \partial y}=-\sin \gamma \quad \gamma(x, 0)=\frac{\pi}{2}=\gamma(0, y),
$$

and $\gamma(x, y) \in(0, \pi)$ for every $(x, y) \in \mathscr{D})$. Thus $\gamma$ is a candidate for the angle between the fibers of a Tchebychev net on $S^{2}$, where $x$ and $y$ are now coordinates with respect to a Tchebychev chart. We must produce an open subset $\mathscr{U}$ of $S^{2}$ which is the domain of this Tchebychev chart.

It is well known that the Riemannian metric can be written on a Tchebychev chart (see $[\mathrm{Pi}],[\mathrm{St}])$ as

$$
g_{(x, y)}=d x^{2}+2 \cos \gamma(x, y) d x d y+d y^{2} .
$$


Since $\frac{\partial}{\partial x}$ and $\frac{\partial}{\partial y}$ have unit magnitude with respect to $g$ on $\mathscr{D}$ it follows that $\mathscr{D}$ endowed with the Riemannian metric $g=d x^{2}+2 \cos \gamma d x d y+d y^{2}$ admits a Tchebychev net $\left(\mathscr{D},\left(\frac{\partial}{\partial x}, \frac{\partial}{\partial y}\right)\right)$. If we can find an open subset $\mathscr{U}$ of $S^{2}$ and an isometry $\varphi$ from $\mathscr{U}$ into or onto $\mathscr{D}$ endowed with $g$, then $(\mathscr{U}, \varphi)$ is a Tchebychev net on $S^{2}$. We will produce such an open subset by using the exponential mapping.

First let us recall some facts from Riemannian geometry.

Let $(M, \rho)$ be a Riemannian manifold and $p \in M$. Then $\exp _{p}$ is a mapping having an open subset of $T_{p} M$ as its domain and $M$ as its codomain, and such that

$$
\exp _{p}(v)=\sigma(1),
$$

where $\sigma$ is the geodesic of $(M, \rho)$ such that $\sigma(0)=p$ and $\dot{\sigma}(0)=v$.

The Riemannian metric $\rho$ induces a unique Riemannian connection $\nabla$ on $M$. Many of the computations needed can be done conveniently by using the Christoffel symbols with respect to some coordinate chart.

Let $\left(U,\left(x^{1}, x^{2}\right)\right)$ be a local coordinate chart on $M$. Then the Christoffel symbols $\left\{\Gamma_{i j}^{k}\right\}_{1 \leq j, k \leq 2}$ are $C^{\infty}$-functions on $U$ defined by

$$
\nabla \frac{\partial}{\partial x^{i}} \frac{\partial}{\partial x^{j}}=\Gamma_{i j}^{k} \frac{\partial}{\partial x^{k}},
$$

where the summation convention is used.

Let $\rho=\rho_{i j} d x^{i} d x^{j}$ with respect to the local coordinates on $U$.

Define $\left\{\rho^{i j}\right\}_{1 \leq i, j \leq n} \subset C^{\infty}(U)$ by

$$
\rho^{i j} \rho_{j l}= \begin{cases}1 & \text { if } i=l, \\ 0 & \text { if } i \neq l .\end{cases}
$$

The Christoffel symbols are easily computed using the formula

$$
\Gamma_{i j}^{k}=\frac{1}{2} \rho^{k l}\left[\frac{\partial}{\partial x^{i}} \rho_{l j}+\frac{\partial}{\partial x^{j}} \rho_{i l}-\frac{\partial}{\partial x^{l}} \rho_{i j}\right] \text {. }
$$

Let $\sigma:[a, b] \rightarrow M$ be a geodesic such that $\sigma([a, b]) \subset U$. Then, writing the components of $\sigma$ with respect to the local coordinates as $\sigma=\left(\sigma^{1}, \sigma^{2}\right)$, we have

$$
\left.\ddot{\sigma}^{i}+\dot{\sigma}^{j} \dot{\sigma}^{k} \Gamma_{j k}^{i}\right)=0, \quad i=1, \ldots, n .
$$

Let us consider the Riemannian manifold $(\mathscr{D}, g)$. Using (3.15) we compute the Christoffel symbols:

$$
\begin{aligned}
& \Gamma_{11}^{1}=\Gamma_{22}^{2}=\cot (\gamma) \gamma y, \\
& \Gamma_{11}^{2}=-\csc (\gamma) \gamma_{x}, \\
& \Gamma_{22}^{1}=-\csc (\gamma) \gamma_{y}, \\
& \Gamma_{12}^{1}=\Gamma_{12}^{2}=\Gamma_{21}^{1}=\Gamma_{21}^{2} \equiv 0 .
\end{aligned}
$$

Remark 3.4. The fact that $\Gamma_{i j}^{k}=0$ for $i=k$ is equivalent to the statement that on a Tchebychev net each fiber field is parallel along the integral curves of the remaining fiber fields. 
Let $t \mapsto(x(t), y(t))$ be a geodesic of $(\mathscr{D}, g)$. Then by (3.16) and (3.17) we get

$$
\begin{aligned}
& \dot{x}=u \\
& \dot{y}=v \\
& \dot{u}=-\cot (\gamma) \gamma_{x} u^{2}+\csc (\gamma) \gamma_{y} v^{2}, \\
& \dot{v}=\csc (\gamma) \gamma_{x} u^{2}-\cot (\gamma) \gamma_{y} v^{2} .
\end{aligned}
$$

The following facts are obtained from Fact 3.2 and Eq. (3.18).

FACT 3.5. The curves

$$
\begin{aligned}
t & \mapsto(t, 0), & & |t| \leq \pi, \\
t & \mapsto(0, t), & & |t| \leq \pi,
\end{aligned}
$$

are both geodesics.

FACT 3.6. If $t \mapsto(x(t), y(t)),|t|<b$, is a geodesic, then the curves

$$
t \mapsto( \pm x(t), \pm y(t))
$$

are all geodesics.

Because of this we consider geodesics in the first quadrant unless such a goedesic crosses the $x$-or the $y$-axis.

FACT 3.7. If $t \mapsto(x(t), y(t))$ is a geodesic, then $t \mapsto(y(t), x(t))$ is a geodesic.

In particular the goedesic $\sigma:[-b, b] \rightarrow \mathscr{D}$ satisfying $\sigma(0)=0$ and $\dot{\sigma}(0)=$ $\left(\frac{1}{\sqrt{2}}, \frac{1}{\sqrt{2}}\right)$ lies on the line $x=y$.

FACT 3.8. $\gamma_{x}(x, y)=-\int_{0}^{y} \cos (\alpha(x, \varphi)) d \varphi$. Thus

$$
0 \geq \operatorname{sgn}(y) \gamma_{x}(x, y) \geq-|y| \quad \text { and } \quad \gamma_{x}(x, y)=0 \quad \text { iff } y=0,
$$

and

$$
0 \geq \operatorname{sgn}(x) \gamma_{y}(x, y) \geq-|x| \quad \text { and } \quad \gamma_{y}(x, y)=0 \quad \text { iff } x=0 .
$$

Lemma 3.9. The domain of $\exp _{0}$ contains $\left(\frac{\delta \pi}{2}\right)\left(\frac{1}{\sqrt{2}}, \frac{1}{\sqrt{2}}\right)$ for some $\delta>1$.

Proof. Let $\sigma:[-b, b] \rightarrow \mathbb{R}^{2}, \sigma(t)=(x(t), x(t))$ be the geodesic satisfying $\sigma(0)=$ 0 and $\dot{\sigma}(0)=\left(\frac{1}{\sqrt{2}}, \frac{1}{\sqrt{2}}\right)$. Let $u(t)=\dot{x}(t)$ for all $t$

$$
1=\|u(t)\|_{g}^{2}=2(1+\cos \gamma) u^{2}(t) .
$$

Since $\gamma(x(t), x(t)) \in\left(0, \frac{\pi}{2}\right)$ we conclude that $0 \leq u(t) \leq \frac{1}{\sqrt{2}}$. Thus $x^{2}(t) \leq\left(\frac{t}{\sqrt{2}}\right)=$ $\frac{t \sqrt{2}}{2}$. Since $\frac{\pi^{2}}{8}<\frac{\pi}{2}$ it now follows that $x\left(\frac{\pi}{2}\right)$ is defined and $(x(t), x(t)) \in \operatorname{Int}(\mathscr{D})$ for all $t \in\left[0, \frac{\pi}{2}\right]$.

We would like to find an isometry from some subset of $S^{2}$ into $\mathscr{D}$ such that the south plole is mapped to the origin. Hence some longitude would be mapped to the geodesic $\sigma$ which we had just considered and in particular $\sigma\left(\frac{\pi}{2}\right)$ would correspond to a point on the equator on $S^{2}$. We now produce as a candidate for the equator a geodesic passing through $\sigma\left(\frac{\pi}{2}\right)$ and orthogonal to $\sigma$.

Lemma 3.10. Consdier the geodesic $\mu$ of $(\mathscr{D}, g)$ such that $\mu(0)=\sigma\left(\frac{\pi}{2}\right)$ and $\dot{u}(0)$ is a unit vector such that $\left(\dot{\mu}(0), \dot{\sigma}\left(\frac{\pi}{2}\right)\right)$ forms a positively oriented orthonormal basis. 
Then $\mu$ can leave $\mathscr{D} \cap\{(x, y): x \geq 0, y \geq 0\}$ by crossing the $x$-axis and no other part of the boundary.

Proof. Let

$$
\mathscr{D}^{+}=\mathscr{D} \cap\{(x, y): x \geq 0, y \geq 0\},
$$

$(\bar{x}, \bar{x})=\exp _{0}\left(\frac{\pi}{2}\left(\frac{1}{\sqrt{2}}, \frac{1}{\sqrt{2}}\right)\right)$, and $\mu(t)=(x(t), y(t)), t \in[0, T]$, where $T$ is chosen such that $\mu(t) \in \operatorname{int}\left(\mathscr{D}^{+}\right)$for all $t \in[0, T]$. Then $\sin (\gamma(\mu(t))$ is bounded away from 0 and $x(t)$ is bounded above for all $t \in[0, T]$. In particular,

$$
x(t) \csc (\gamma(\mu(t))) \quad \text { is bounded on [0,T]. }
$$

Now consider $u$ and $v$ as in Eq. (3.18). By Fact 3.8 we obtain,

$$
\dot{v}(t)<x(t) \cot (\gamma(\mu(t))) v^{2} .
$$

Hence $-\frac{1}{v(t)}+\frac{1}{v(0)} \leq \int_{0}^{t} x(\tau) \cot \gamma(\mu(\tau)) d \tau<\infty$ for any $t \in[0, T]$. Thus $v(t)$ never changes sign in $[0, T]$ and since $v(0)<0, v(t)<0$ for all $t \in[0, T]$. Therefore,

$$
y(t)<y(0)=\bar{x} \quad \text { for all } t \in(0, T] .
$$

Also by Fact 3.8,

$$
\frac{d}{d t}(u(t)+v(t))=\gamma_{x} \frac{(1-\cos \gamma)}{\sin \gamma} u^{2}+\gamma_{y} \frac{(1-\cos \gamma)}{\sin \gamma} u^{2}<0 \quad \text { for } t \in[0, T] .
$$

Since $u(0)+v(0)=0$, it follows that

$$
u(t)+v(t)<0 \quad \text { for all } t \in[0, T] .
$$

Now the conditions $v(t)<0$ and $u(t)+v(t)<0$ for all $t \in(0, T]$ imply that the curve $\mu(t),(t \in(0, T])$ is in the triangle $\Delta$, bounded by the lines

$\{(x, 0): 0 \leq x \leq \sqrt{2} \bar{x}\}, \quad\{(x, x) \in 0 \leq x \leq \bar{x}\} \quad$ and $\quad\{(x, 2 \bar{x}-x): \bar{x} \leq x \leq 2 \bar{x}\}$.

Since the line $\{(x, 2 \bar{x}-x): 0 \leq x \leq 2 \bar{x}\}$ is in the interior of $\mathscr{D}^{+}, \mu$ does not leave $\mathscr{D}^{+}$by crossing the curve $x y=\frac{\pi}{2}$ or $y=\pi$.

Moreover, the curve $\mu$ does not cross the line $\{(x, x): 0 \leq x \leq \bar{x}\}$ for $t \in[0, T]$, since if it does, there is a geodesic triangle contained in $\Delta$ the sum of whose interior angles is greater than $\frac{\pi}{2}$. However, since the Gaussian curvature of $g$ is equal to 1 and since the measure of $\Delta$ (with respect to the measure induced by $g$ ) is less than 1 , this contradicts the Gauss Bonnet theorem.

Since $v(t)<0, t \in[0, T]$, it follows that the geodesic $\mu$ ultimately leaves $\mathscr{D}^{+}$ by crossing the $x$-axis.

Let $\tilde{\mathscr{D}}$ be the closed region bounded by the line $x=y$, the geodesic $\mu$, and the positive $x$-axis.

We now show that we can construct a diffeomorphism of a segment of the hemisphere into $\tilde{\mathscr{D}}$. We introduce Gauss coordinates to do so.

LEMMA 3.11. The map

$$
\psi:\left[0, \frac{\pi}{4}\right] \times\left[0, \frac{\pi}{2}\right] \rightarrow \tilde{\mathscr{D}}
$$

defined by

$$
\psi(\theta, t)=\exp _{0}(t(\cos \theta, \sin \theta))
$$

is well defined, and it is the inverse of a Gauss coordinate system on the sector $\tilde{\mathscr{D}}$. 
Proof. Let $\mu$ be the geodesic constructed in Lemma 3.10

Thus we wish to show that all geodesics in $\tilde{\mathscr{D}}$ which start radially from the origin meet $\mu$ orthogonally; i.e., that, $U:=\left\{\theta \in\left[0, \frac{\pi}{4}\right]: \psi\left(\theta,\left[0, \frac{\pi}{2}\right]\right) \in \mathscr{D}, \psi\left(\theta, \frac{\pi}{4}\right) \in \mu\right.$, and $\frac{\partial}{\partial t} \psi\left(\theta, \frac{\pi}{2}\right)$ is orthogonal to $\left.\mu\right\}$ is all of $\left[0, \frac{\pi}{4}\right]$.

Clearly $\frac{\pi}{4} \in U$. Let $\theta_{0} \in U$. Then the map $t \mapsto \psi\left(\theta_{0}, t\right), t \in\left[0, \frac{\pi}{2}\right]$ is a geodesic by definition. Also by our construction the curvature on $\tilde{D}$ is 1 and hence for each $t_{0} \in\left[0, \frac{\pi}{2}\right]$ there exists an isometry from a neighborhood of $\psi\left(\theta_{0}, t_{0}\right)$ in $\tilde{\mathscr{D}}$ onto an open subset of the standard sphere. It follows from the compactness of $\left[0, \frac{\pi}{2}\right]$ that there exists an open subset $V$ of $\theta_{0}$ in $\left[0, \frac{\pi}{4}\right]$ such that $\psi\left(V \times\left[0, \frac{\pi}{2}\right]\right)$ is isometric to a triangular sector around $\psi\left(\theta_{0} \times\left[0, \frac{\pi}{2}\right]\right)$ of the standard sphere with a vertex at the south pole and a base on the equator. Now $\psi\left(V \times \frac{\pi}{2}\right)$ is mapped onto the equator, thus $V \subset U$. Therefore $U$ is an open subset of $\left[0, \frac{\pi}{4}\right]$. Since $U$ is closed by the continuity of $\psi, U=\left[0, \frac{\pi}{4}\right]$. In particular, $\psi$ is defined and $\operatorname{Rng}(\psi) \subset \tilde{\mathscr{D}}$. Since $\psi\left(0 \times\left[0, \frac{\pi}{2}\right]\right)$ is a subset of the horizontal axis and $\psi\left(\frac{\pi}{4} \times\left[0, \frac{\pi}{2}\right]\right)$ is along the line $x=y$ and $\psi\left(\left[0, \frac{\pi}{4}\right] \times \frac{\pi}{2}\right)$ contains $\mu$, it follows that $\psi\left(\left[0, \frac{\pi}{4}\right] \times\left[0, \frac{\pi}{2}\right]\right)$ contains $\tilde{\mathscr{D}}$. We claim that $\psi$ is one to one. In order to show this we use the following fact from [Kl]. Let $p \in M$ where $M$ is a Riemannian manifold, and let $r(p)=\sup _{r \in \mathbb{R}}\left\{r \geq 0\right.$ such that exp: $B_{r}(0) \subset T_{p} M \rightarrow M$ is injective $\} .(r(p)$ is called the injectivity radius at $p$.) Then $\exp _{p}: B_{r(p)}(0) \subset T_{p} M \rightarrow M$ is a diffeomorphism onto some open set (and this is a Gauss coordinate system).

Now consider $\varphi: \overline{B_{\pi / 2}(0)} \rightarrow \mathscr{D}$ given by

$$
\varphi(\theta, t)=\exp _{0}(t(\cos \theta, \sin \theta))
$$

where $(t, \theta)$ are polar coordinates on the disc. If $\varphi$ is not one to one, then the injectivity radius is less than or equal to $\frac{\pi}{2}$. It follows that there are 2 geodesics $\eta_{1}, \eta_{2}$ in $\mathscr{D}$ eminating from the origin of the same length $r(0) \leq \frac{\pi}{2}$ which meet. Let the parameter $t$ denote arclength parameterization on each geodesic. Since $\exp _{0}: B_{r(0)}(0) \rightarrow \mathscr{D}$ is the inverse of a Gauss coordinate system, we have an isometry from $\exp _{0}\left(B_{r(0)}(0)\right)$ to a southern polar cap of radius $r(0)$ of the sphere. By considering the isometry, we conclude that the distance $d\left(\eta_{1}(t), \eta_{2}(t)\right)$ is increasing with $t$.

But this is a contradiction since $\eta_{1}(r(0))=\eta_{2}(r(0))$. Since $\varphi$ is one to one it follows that $\psi$ is one to one. We have now seen that the injectivity radius at the origin is greater than $\frac{\pi}{2}$ and hence $\psi$ is a diffeomorphism providing a sectorial Gauss coordinate system.

We have now established that $\psi:\left[0, \frac{\pi}{4}\right] \times\left[0, \frac{\pi}{2}\right] \rightarrow \tilde{\mathscr{D}}$ is a bijection. Also, no point in $\tilde{\mathscr{D}}$ is antipodal to the origin along any geodesic. Hence $\psi^{-1}$ is a Gauss coordinate system on $\tilde{\mathscr{D}}$ and therefore $\tilde{\mathscr{D}}$ can be mapped isometrically onto the subset of $S^{2}$ in the closed southern hemisphere bounded between longitudes 0 and 90 degrees.

By symmetry there exists an isometry from the closed southern hemisphere into $\mathscr{D}$ such that the image is contained in the interior of $\mathscr{D}$. Since Gauss coordinates have open domain, we can increase the domain slightly and obtain a surjective isometry $\varphi: W \longrightarrow \hat{W}$ such that $W$ is an open subset of $S^{2}$ and contains the closed southern 
hemisphere and $\hat{W}$ is an open subset of $\mathscr{D}$. Moreover, we can use the inverse of $\varphi: W \rightarrow \mathbb{R}^{2}$ to construct a Tchebychev net on $S^{2}$. This concludes the proof of the Main Theorem.

Acknowledgment. I would like to thank my Thesis advisor W. O. Williams for the many hours he spent working with me on this result.

\section{REFERENCES}

[Tch] P. L Tchebychev, Sur la coupe des vêtements, Assoc. franç. pour l'avancement des sci., Congrés de Paris, 154-155 (1878)

[Bie] L. Bieberbach, Über Tchebychefsche Netz auf Flächen negative Krümmung, S-b Preuss. Akad. Wiss., Phys. Math. Kl. 23, 294-321 (1926)

[Haz] J. N. Hazzidakis, Über einige Eigenschaften der Flächen mit constantem Krümmungsmass, J. Reine und Angew. Math. (Crelle's J.) 88, 68-73 (1880)

[Kl] W. Klingenberg, Riemannian geometry, Lectures on Closed Geodesics, Springer-Verlag, BerlinHeidelberg-New York, 1978

[Pi] A. C. Pipkin, Equilibrium of Tchebychev nets, Arch. Rational Mech. Anal. 1, 81-97 (1984)

[Ser] M. Servant, Sur l'habillage des surfaces, C. R. Acad. Sci. 137, 112-115 (1903)

[Sa1] S. Samelson, Generalized Tchebychev nets, M. Sc. Thesis, Department of Mathematics, Carnegie Mellon University, 1986

[St] J. J. Stoker, Differential Geometry, Wiley, New York, 1969 